\section{BiCMOS variable gain transimpedance amplifier for automotive applications}

T. De Ridder, P. Ossieur, X. Yin, B. Baekelandt, C. Mélange, J. Bauwelinck, X.Z. Qiu and J. Vandewege

A new BiCMOS variable gain transimpedance amplifier with a large area integrated photodiode for automotive applications is presented. Through careful control of the input pole position and the frequency response of the core amplifier, the bandwidth of the transimpedance amplifier varies from 112 to $300 \mathrm{MHz}$ when its gain changes from $14.2 \mathrm{k} \Omega$ to $400 \Omega$. The proposed circuit configuration maintains a high voltage across a common anode photodiode, and its bandwidth in highest gain varies from 121 to $102 \mathrm{MHz}$ over a temperature range of -40 to $+140^{\circ} \mathrm{C}$. Simulation results in a $0.6 \mu \mathrm{m} \mathrm{Si}$ BiCMOS technology are given. The amplifier consumes $16 \mathrm{~mW}$ from a $3.3 \mathrm{~V}$ supply.

Introduction: Optical transmission technology is increasingly used in cars as an alternative for copper-based solutions [1]. Optical receivers for automotive communication networks must cope with a high ambient temperature range $\left(-40\right.$ to $\left.+125^{\circ} \mathrm{C}\right)$, be cost-effective and low power. Cost-effectiveness implies usage of an integrated photodiode (PD) with large diameter ( $430 \mu \mathrm{m}$ in this Letter) to relax the mechanical alignment accuracy requirement, resulting in a high PD capacitance of $4.8 \mathrm{pF}$. PDs can be made using the $p$-substrate as an anode and an $n^{+}$ contact as a cathode [2]. To ensure a fast PD and minimise its capacitance, its reverse bias must be high. This is challenging at a supply voltage of $3.3 \mathrm{~V}$. Variable gain transimpedance amplifiers (TIAs) are needed to ensure high dynamic range. In [3], a variable gain TIA is realised by splitting the core amplifier into two parallel amplifiers and adding their outputs together in a weighted sum fashion. Variable gain was realised by adjusting the weighting factor. However, owing to the varying open-loop gain it is difficult to control the bandwidth and peaking of the frequency response. In [4-6], current switches at the input of the TIA are used to steer part of the photodiode current away from the TIA for high optical input power. However, the voltage drop across the current switches results in low bias voltage across a common anode PD. In [7], a Darlington input stage is combined with MOSFETs used as voltage-controlled resistors to achieve wide dynamic range. However, the Darlington input stage is not suitable for a supply voltage of $3.3 \mathrm{~V}$, and the use of ten MOSFETs to control the gain and frequency response makes the circuit design over a wide temperature range difficult. In this Letter we propose a new circuit that overcomes these disadvantages, and can handle a junction temperature range of -40 to $+140^{\circ} \mathrm{C}$.

Transimpedance amplifier: As shown in Fig. 1, the TIA is fully differential. Balanced operation is achieved using a matched dummy PD. The optical signal is focused upon the real PD. In the automotive environment, a differential TIA offers many advantages over a single-ended TIA despite increased die size and input noise current. It has high immunity against common-mode (CM) and supply noise, which is important given the high levels of electromagnetic interference in cars. Immunity against CM noise is also required in case a charge pump is used to increase bias voltage across the PD [8]. Furthermore, the loop gain characteristics (set by the differential response) can be determined independently from the DC-biasing voltages, which is advantageous over implementations such as in [7] given the required temperature range. The increased input current noise is not a problem since transmission distances in the car are low and utmost sensitivity is not needed. As can be seen in Fig. 1, the first stage of the core amplifier consists of a resistively loaded differential pair $\mathrm{Q}_{1}, \mathrm{Q}_{2}$. Tail current $\mathrm{l}_{1}$ is proportional to absolute temperature to ensure stable behaviour over temperature. Emitter followers $\mathrm{Q}_{1 \mathrm{~A}}, \mathrm{Q}_{1 \mathrm{~B}}, \mathrm{Q}_{2 \mathrm{~A}}$ and $\mathrm{Q}_{2 \mathrm{~B}}$ provide a $\mathrm{CM}$ level shift and low drive impedance for the next stage. A second differential pair $\mathrm{Q}_{3}, \mathrm{Q}_{4}$ is added to obtain high loop gain despite the low supply voltage of $3.3 \mathrm{~V}$. This ensures that the bandwidth of the TIA is sufficiently high despite the large PD capacitance. Emitter feedback resistors were added to stabilise gain as a function of temperature. Capacitors are connected across these resistors to create zeros to compensate for the input poles of $\mathrm{Q}_{3}, \mathrm{Q}_{4}$. The tail current of the differential pair is set in a CM feedback loop which fixes the CM output voltage of the TIA to $1.8 \mathrm{~V}$. This ensures that bias voltage across the PD remains high, independent of the TIA gain. As two inverting stages are used, negative feedback requires an additional sign-inversion. This is done by swapping the TIA output phases. Variable gain is realised by shunting the feedback resistor with MOSFETs $\mathrm{M}_{3 \mathrm{~A}}, \mathrm{M}_{3 \mathrm{~B}}$. As reducing the feedback resistance moves the dominant pole to higher frequencies, care must be taken to ensure stability. This is done by reducing the gain of both differential pairs using MOSFETs $\mathrm{M}_{1}$ and $\mathrm{M}_{2}$. This moves the collector poles to higher frequencies, and does not disturb the DC-bias points. It only requires two MOSFETs to control the gain of the core amplifier whereas in [7] seven MOSFETs were needed. This results in better control of the circuit over temperature. Furthermore, capacitance is added to the input of the TIA using MOSFETs $\mathrm{M}_{4 \mathrm{~A}}, \mathrm{M}_{4 \mathrm{~B}}$. Together with the reduction of the open-loop gain, this reduces the bandwidth enlargement when the TIA gain is reduced. Other implementations use a Miller capacitance connected across the TIA [9]. However, this would have resulted in a capacitor of a few tens of femtofarad, the process variations of which would be too large. $\mathrm{C}_{1}$ is realised as a stacked poly-metal capacitor of $3.45 \mathrm{pF}$. As the area of this capacitor $\left(40 \times 70 \mu \mathrm{m}^{2}\right)$ is small compared to the PD area this is an acceptable solution.

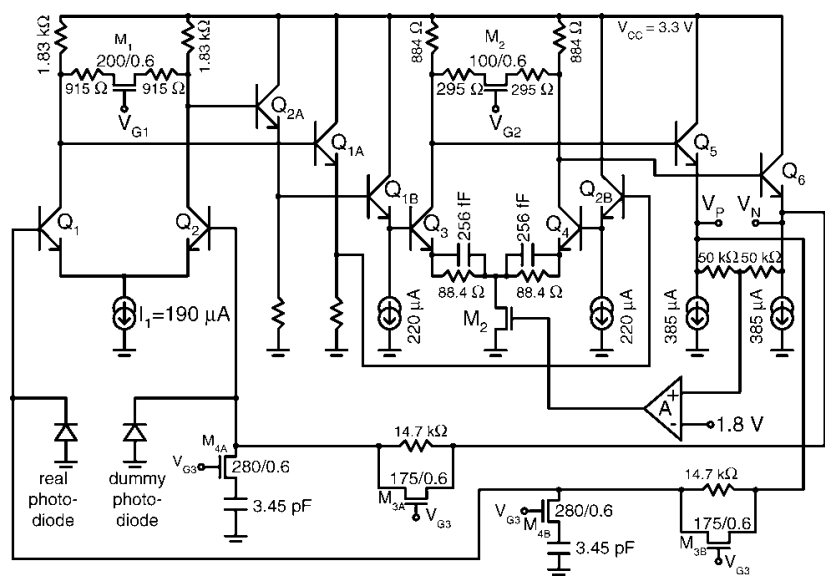

Fig. 1 Proposed variable gain transimpedance amplifier

Automatic gain control loop: Fig. 2 shows the automatic gain control (AGC) loop, which adjusts the gain so that the differential output swing equals a reference $2 \mathrm{~V}_{\mathrm{REF}}$. The output swing of a single phase of the TIA is detected using a positive and negative peak detector. The outputs of these peak detectors are fed to transconductor $G_{M}$ with built-in DC-offset $\mathrm{V}_{\mathrm{REF}}$. Hence, when the AGC-loop has reached equilibrium, the swing of a single phase of the TIA equals $V_{\text {REF }}$. The value of $\mathrm{V}_{\mathrm{REF}}$ is not critical as long as the output swing can be handled without significant pulse width distortion (PWD). $\mathrm{V}_{\mathrm{REF}}$ is generated by sizing the input MOSFETs of the transconductor differently. The output of the transconductor drives the gates of MOSFETs $M_{3 A}, M_{3 B}$ and $\mathrm{M}_{4 \mathrm{~A}}, \mathrm{M}_{4 \mathrm{~B}}$ (corresponding to $\mathrm{V}_{\mathrm{G} 3}$ in Fig. 1). To ensure that the rate of change of the on-resistance of all MOSFETs is equal, their gate-source voltages $\mathrm{V}_{\mathrm{GS}}$ must be equal. The additional circuitry shown in Fig. 2 ensures that $\mathrm{V}_{\mathrm{G} 1}$ and $\mathrm{V}_{\mathrm{G} 2}$ are such that the $\mathrm{V}_{\mathrm{GS}}$ of $M_{1}$ and $M_{2}$ tracks the $V_{G S}$ of $M_{3 A}, M_{3 B}$ and $M_{4 A}, M_{4 B}$.

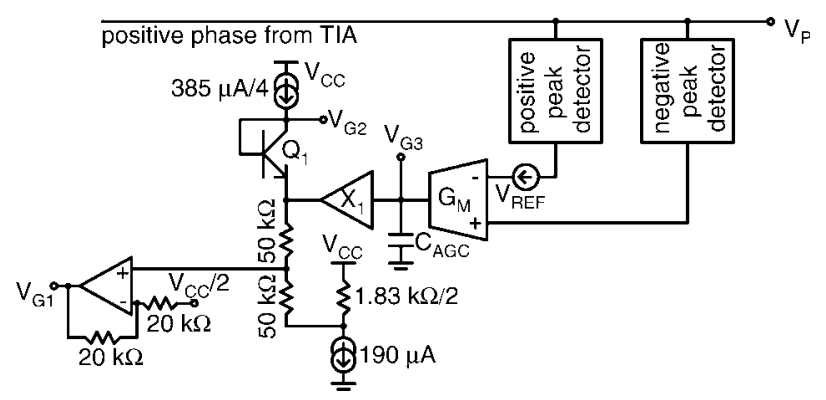

Fig. 2 Automatic gain control loop

Simulation results: All simulation results were performed on the schematics back annotated with the interconnection capacitance. Fig. 3 shows the output voltage and PWD against input current. $\mathrm{V}_{\mathrm{REF}}$ was designed to be $125 \mathrm{mV}$. At a PWD of $10 \%$, an input current of 
$750 \mu \mathrm{A}$ can be handled. For a PD reponsivity of $0.4 \mathrm{~A} / \mathrm{W}$, this corresponds to an average optical power of $-0.3 \mathrm{dBm}$. Fig. 4 shows the differential gain of the TIA against gain control voltage $\mathrm{V}_{\mathrm{G} 3}$, as well as its $3 \mathrm{~dB}$ bandwidth against $\mathrm{V}_{\mathrm{G} 3}$ and temperature. No peaking in the frequency response was observed over the entire gain range. Even though bandwidth increases with decreasing TIA gain, this is not problematic as additional filtering can be added in the post-amplifier following the TIA. At highest gain, the TIA bandwidth changes from 102 to $121 \mathrm{MHz}$ over a temperature range of -40 to $140^{\circ} \mathrm{C}$. At a temperature of $140^{\circ} \mathrm{C}$ (the worst case), the input-referred noise current is $108 \mathrm{nA}_{\mathrm{rms}}$. A sensitivity of $-28.0 \mathrm{dBm}$ can be estimated at a bit rate of $150 \mathrm{Mbit} / \mathrm{s}$ with a bit error rate of $10^{-9}$. Sensitivity and overload exceed typical automotive requirements by a large amount [1].

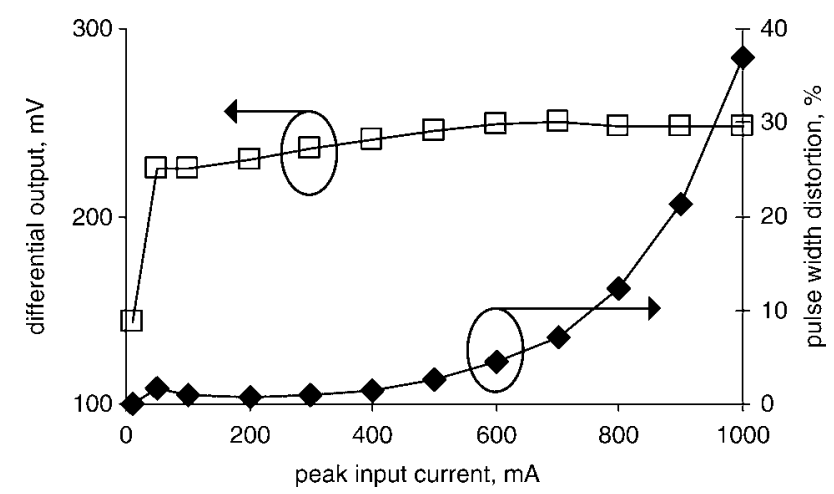

Fig. 3 Output voltage and pulse width distortion

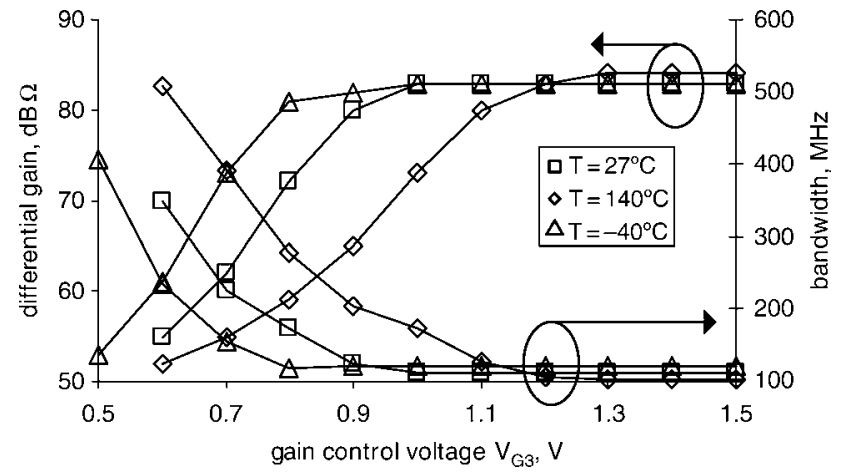

Fig. 4 Differential gain and bandwidth
Conclusion: We present a new TIA with variable gain suitable for automotive applications. Compared to previous configurations, the proposed TIA maintains stable bandwidth both over the temperature range -40 to $+140^{\circ} \mathrm{C}$, and over its full gain range, and combines this with a stable and high bias voltage across a common anode PD.

(C) The Institution of Engineering and Technology 2008

28 October 2007

Electronics Letters online no: 20083101

doi: 10.1049/el:20083101

T. De Ridder, X. Yin, B. Baekelandt, C. Mélange, J. Bauwelinck, X.Z. Qiu and J. Vandewege (Ghent University, INTEC/IMEC, Gent, B-9000, Belgium)

E-mail: tine.deridder@intec.ugent.be

P. Ossieur (FWO-Vlaanderen, Belgium)

\section{References}

1 Kibler, T., Poferl, S., Bock, G., Huber, H.P., and Zeeb, E.: 'Optical data buses for automotive applications', J. Lightwave Technol., 2004, 22, (9), pp. 2184-2199

2 Swoboda, R., and Zimmermann, H.: 'A 2.5-Gb/s receiver OEIC in $0.6 \mu \mathrm{m}$ BiCMOS technology', IEEE Photonics Technol. Lett., 2005, 16, pp. $1730-1732$

3 Yamashita, K., Kinoshita, T., Takasaki, Y., Maeda, M., Kaji, T., and Maeda, N.: 'A variable transimpedance amplifier for use in wide dynamic range optical receivers', IEEE J. Solid-State Circuits, 1986, 21, pp. 324-330

4 Vandenbroeke, L.A.D., and Nieuwkerk, A.J.: 'Wide-band integrated optical receiver with improved dynamic range using a current switch at the input', IEEE J. Solid-State Circuits, 1993, 28, pp. 862-864

5 Ruotsalainen, T., Palojarvi, P., and Kostamovaara, J.: 'A current-mode gain-control scheme with constant bandwidth and propagation delay for a transimpedance amplifier', IEEE J. Solid-State Circuits, 1999, 34, pp. $253-258$

6 Chen, R.Y.: 'A CMOS infrared wireless optical receiver front-end with a variable-gain fully-differential transimpedance amplifier', IEEE Trans. Consum. Electron., 2005, 51, pp. 424-429

7 Meyer, R.G., and Mack, W.D.: 'A wideband low-noise variable-gain BiCMOS transimpedance amplifier', Electron. Lett., 1992, 28, (23), pp. 2127-2130

8 Swoboda, R., Knorr, J., and Zimmermann, H.: 'A 5-Gb/s OEIC with voltage up-convertor', IEEE J. Solid-State Circuits, 2005, 40, (7), pp. $1521-1526$

9 Das, M.B., Chen, J.W., and John, E.: 'Designing optoelectronic integrated circuit (OEIC) receivers for high sensitivity and maximally flat frequency response', J. Lightwave Technol., 1995, 13, pp. $1876-1884$ 